\title{
English Irony and Word Play through Drama: New Approach to Mastering Discourse
}

\author{
Anna Gornostaeva, Vera Beliaeva \\ Moscow State Linguistic University \\ Russian State Humanitarian University
}

\begin{abstract}
The English culture and communicative style are closely connected with irony, while the English language is rich in metaphors, homonyms and homophones, ironic comparisons and ironic word play. It has been noted, that, being an integral part of communication, irony is vital for the English as a way to perceive reality and an instrument to create discourse [1, 2]. Yet, the question of teaching irony and other expressive means is still open. The current study is dedicated to teaching English as a foreign language to students of intermediate level, focusing on lexis and phraseology, namely such expressive means as irony and word play. The suggested method is based on analyzing pieces of English drama, concentrating on interesting linguistic phenomena, which means studying irony and word play as textual categories. The next step involves staging performances, which includes using these phenomena as categories of discourse. The combination of the two approaches ensures a good effect in education. The paper gives a detailed description of the method and its advantages.
\end{abstract}

\section{Introduction}

Modern world and development of new technologies pose great challenges for teachers as well as students. There is a great many methods and trends in teaching foreign languages being introduced into practice both for children and grownups; still, there is no universal recipe suitable for any kind of target audience. We are facing the situation where a need of involving pupils in the communicative process is urgent. By achieving it, the learners could deeply immerse in the process of communication. One of the means to reach this aim is combining traditional teaching practice with elements of theatre play; thus, setting the goal of staging a performance in a foreign language, pupils concentrate on reaching it, simultaneously acquiring lexical and grammar material.

Drama is one of the modern trends in teaching a foreign language. The combination of a foreign language class with theatre appears to be an interesting and exciting approach. The real aim of the process - acquiring linguistic skills - is disguised under a set of tasks, involving studying the screenplay, analyzing and learning the text, playing the role and, finally, staging the performance. While fulfilling these tasks the students are involved in a play, feel more relaxed than at usual classes and have motivation. All these positive factors contribute to creating a friendly inspiring atmosphere and to the final effect.

While playing a certain part, young actors face the necessity to become another personality, acquire the logics and feelings of their character. To do it successfully, it is necessary to find "the grain of the role" [7], which is a number of peculiarities, making it possible for an actor to "live" in the suggested circumstances and, moreover, even beyond the role, in any situation that might occur in real life. Trying to impersonate a certain character, an actor puts on not only a new appearance, but a new mind. Thus, the lines of a play become inherent for a student and help to acquire the language naturally.

\section{Methodology}

Recently there have emerged new methods on teaching English through drama for both adult students and children $[3,4,5,6]$, which are aimed at developing various language competences, like speaking skills, listening comprehension, the development of grammatical and lexical mastership. This paper is devoted to teaching irony and wordplay to secondary school pupils (pre-intermediate and intermediate level). Since irony is an important integral feature of the British communicative style [1], it is essential to attract the attention of pupils to these expressive means of the language from an early stage of education. Irony is a category of discourse, ironic word play can be found in dialogues. At the same time these phenomena exist in written texts and should be studied as intertextual category. In this view analyzing pieces of drama followed by staging them seems to be an efficient method, which combines text analysis with discourse analysis. The process of performance involves pupils in discourse, at the same time they follow the script and practice certain language means and devices, learn the mechanisms and functions of English irony.

The present method relies on the works of $\mathrm{G}$. Tovstonogov [7], who accentuated the importance of 
the text for the actors, and his theory of absorbing the lines, penetrating into the implicit meaning. The essence of this method comprises several steps of analyzing the text of a play.

1. It is necessary to single out the most dramatic events in a play and divide the text accordingly into several parts.

2. Distinguish in these events a chain of conflicts (with one's own self, with other people, with circumstances).

3. Find a concrete action in these conflicts (usually it is represented by an active confrontation).

According to G. Tovstonogov, to denote all these things one should concentrate on the main situation and distinguish the conflict behind emotions. It should be noted, that the conflict is not necessarily straightforward, but can be felt subconsciously, through growing suspense.

The understanding of the conflict is absolutely essential for the correct interpretation of the part. Even if the situation is seemingly calm and peaceful, there must be some growing tension. It manifests itself through action, and the main event in a play is the one that inspires the characters to act.

The method of Tovstonogov relied a great deal on movements: while reciting the lines, it is important to emphasize the meaning not only with appropriate intonation, but also accompany the words by movements, gestures, which correspond to the idea.

The character of a movement should conform to the psychological background of the scene. One and the same movement can be done in different ways irritably, angrily, complacently, quietly etc. The information about the nature of each gesture is prompted by the text itself and it is up to an actor to penetrate into the hidden sense and interpret it correctly.

The basics of theatre art is based on the system of the great theatre actors and directors, such as K. Stanislavski, V. Meyerhold, and M. Chekhov, which includes exercises for breathing, movements, body language, speech practice. Theatre play requires a lot of preliminary work, such as the art to breathe properly, the art to speak loudly and distinctly, movements and body posture skills. Since the performance is in English, a lot of attention is paid to phonetics (pronunciation and intonation). Before stage playing, a lot of reading aloud takes place, as well as training lexical and grammar constructions.

\section{Screenplay}

The actors, who are supposed to take part in staging a play, are secondary school children ranging in age from 8 to 12 . At this age children are eager to play and are fond of games, but they are adult enough to expect some result to ensue from their activity. If the aim of studying is formulated as "acquiring certain lexical and grammar material", it is not motivating enough. But, setting a challenging but feasible goal is the best way to make the young learners interested in the process of studying. Moreover, at this age people start feeling the desire to express themselves and identify their personality. From psychological point of view, putting on a mask and impersonating another character is an effective way to set free one's own self and reveal the suppressed feelings and emotions. In this view, pupils are excited to realize their potential on the stage and at the same time create a final product the performance, which is to be shown to the audience at the end of the course. The performance itself is a kind of final exam, which can be taken only once, there is no chance to take it again and improve the mark. So it provides additional motivation to the pupils. The teacher, in his/her turn, pursues one more aim, disguised from the pupils, that is to make them master a certain amount of the foreign language and culture: grammar and lexical material, history, communicative values.

The initial step to staging a performance is choosing the suitable plot. It is a crucial task which accounts for the success of the preparatory process and the final result. While choosing the text for the performance, it was necessary to bear in mind the following factors:

1. The authenticity of the text

2. Substantial number of expressive means, used in the text

3. Number of characters, involved in the action

4. The level of difficulty

5. The age of the actors.

Regarding all the conditions, the choice was limited by "The importance of being Earnest" (O. Wilde), "Pigmalion" (B. Shaw) and "Alice in Wonderland" (L. Carrol) [8]. In view of the pupils' age the latter option was preferred, as a fairy-tale with elements of mysticism is warmly received by children. The text has a substantial amount of language material, based on wordplay, homonyms and homophones, irony etc. This play is a challenge; it has a capturing plot and children feel intrigued and excited to try it, penetrate into the hidden meaning and convey the author's ideas on the stage.

Genre is an essential point, which cannot be ignored. It represents the way of reflecting reality, the angle, at which the author views the reality. The director must take the genre into account while inserting some additional text and scenes into the original play. According to Tovstonogov, the difference between genres is manifested in the range of "suggested circumstances" [7].

In this context, the director's task is: 
1. To pay attention to other genre-markers, not only the division into poem or prose. The length of the lines must be taken into account.

2. To understand the author's view on the situation and life in general, the feeling and emotions, which are necessary to convey.

3. To penetrate into the reasons of the characters' acts and the motives that made the author write the text.

So, the first stage of work consisted of transforming the text into a play and adapting it to the level of the pupils. This process involved omitting several parts of the story, shortening the plot to several scenes and adapting the text, including as many expressive means as possible.

The second stage is presenting the text to the class, reading it aloud, singling out difficult parts, translating and explaining the meaning. According to the method of G. Tovstonogov, each play is a lock, to which the director should find the key [7]. The first reading must take place in a quiet comfortable atmosphere. One must be careful with the first impression, as it might be full of stereotypes and clichés. But Tovstonogov recommends to write down the first impression (e.g. "it is stifling") and after the performance is ready it is desirable to compare the initial picture with the result. The first impression reflects the novelty of feelings, as well as associations with something already seen or felt. That is why it is not recommended to start working at a part immediately at the stage of the first reading. Some more preparation is required.

Each actor, considering his/her part must ask himself/herself a question: what my character and I have in common? What is the difference between us? These questions will lead to the appearance of intellectual distance, which is inevitable in the process of understanding the role.

After distributing the parts each actor concentrates on his/her words, learning them by heart, pronouncing the text with a proper intonation, receiving a feedback from the partner. It is desirable to change parts several times so that each pupil should fully understand and learn the whole text.

While the lines are pronounced on the stage, the dialogue is accompanied by movements, gestures and mimics, which all contributes to better understanding and memorizing the mechanisms of irony.

\section{Preparation and Exercises}

The actual work in a team starts with testing the target audience, which takes place in the form of games, questions-and-answers exercises, short role plays etc. The aim of this stage is to denote the level of foreign language knowledge on the one hand and revealing the children's communicative skills, on the other. The teacher makes acquaintance with the group and the children are involved in communication, which exposes the temperament of the pupils, their abilities to react and their willingness to be engaged in playing. Simultaneously, the preparation period ensures a warm and friendly atmosphere, where people feel free to express their ideas and emotions. Before introducing the text of the chosen play to the class, it is required to go through various short scenarios, which presuppose different situations from real life and are connected with expressing all sorts of emotions, like joy, surprise, amazement, grief, anger etc. Children have a go at conveying genuine emotions, develop communicative skills and reveal their personality This exercise helps them to get prepared for acting on stage and assuming certain roles. At the same time the pupils acquire lexical material which helps them to express themselves: words, phrases, ready-made constructions. Normally it is easily acquired because it is related to real life situations.

Another type of exercises is based on establishing contact with an interlocutor: these include greetings, thanks, requests, apologies etc. This step is very important from the point of building up team spirit and creating mutual understanding among future actors. At the same time, a lot of useful learning is involved in the process which contributes to pupils' mastering the language and developing interpersonal skills.

Various types of exercises, connected with moving, speaking, interacting, expressing oneself, usually receive a warm welcome from the child audience; they seem like a game, but at the same time they serve a definite aim of preparing actors for a more important and difficult work.

The exercise period may last from several hours to several weeks, depending on the level of the audience. It includes various types of activities and is aimed at warming up the pupils and getting them ready for playing on the stage. When the actors are ready the text of the play is introduced. It should be adapted according to the pupils' age, interests, abilities and level of language difficulty and comprise a lot of dialogues and conversations.

The work with the screenplay starts from reading the text aloud in parts; everyone should have a chance to impersonate different characters, try different intonation patterns, expressing various meanings. This step is mostly connected with language work, for the text of the play needs deep understanding before being pronounced from the stage. Lexical and grammar material is studied, analyzed and trained. Pupils are asked not only to reproduce lines from the screenplay, but also to think of their own examples in similar situations. 
The process of studying the screenplay takes long and may be quite tiresome; the mission of the teacher here is to support interest in his/her pupils and to mix learning with exercises directed to activity and movement.

Another important mission of the teacher (and, simultaneously, the director of the play) is to distribute roles to the actors. Normally, every pupil would like to play the leading part, but there are secondary roles, which have to be covered as well. It is essential to convince the young actors that all the characters deserve equal attention and each part, even if it consists of several lines, contributes to the overall picture. The young actors should create their own understanding of the character they impersonate, see it not as a static figure, but as a living being in the process of formation and development. So, it becomes clear that every role should be acted with force and diligence, to attract the attention of the audience and create the desired effect. It is crucial to take into consideration that all the lines are connected with each other, which means that actors should learn and analyze the whole text, not just their part separately.

After the parts in the play are distributed, theatre rehearsals begin. The culmination - the performance on the stage - is a result of diligent work, cooperation and mutual efforts, which contribute to developing children's knowledge and skills.

\section{Case Study and Commentary}

As it was already mentioned, the script of the play is prepared in advance. It should include a considerable amount of lexical, grammatical and cultural material which is recommended for studying. In this paper the attention is paid to expressive means that are widely represented in the chosen play. Here are some examples from the screenplay that are recommended for analyzing.

\section{$\underline{\text { Situation } 1}$}

Alice and the narrator (L. Carrol) are at picnic, talking to each other. L. Carrol is speaking about his childhood, but Alice is not listening - she is watching a white rabbit calling her. Deep in her thoughts, she is considering a serious question, which determines the beginning of this mysterious story.

Alice: "To go or not to go? To go or not to go?"

This is an allusion to the famous "To be or not to be" - the words of Hamlet, the character of one of the best tragedies of Shakespeare. This phrase, together with some comments of the teacher, gives children some knowledge about one of the most significant authors in the history of theatre and drama. Alice's question - whether to follow the mysterious way or to stay - may be ironically compared to the idea of Hamlet on the sense of life. In "Alice in Wonderland" the answer to this dramatic question turns out to be a positive one, which gives a start to the exciting plot.

\section{$\underline{\text { Situation } 2}$}

After eating a piece of magic pie and sipping some magic drink, Alice is getting larger and then smaller. At this moment she is giving a small monologue, wondering at the changes that are happening to her.

Alice: "Curiouser and curiouser!"

In the original there are no exact words like these, here we use deliberate quotation spoofing, which can be regarded as "childish" language. It is reflected in the Russian translation of the book and the phrase is familiar to almost everybody. The aim of such transformation is to make children interested in the text, to make it funny for them. At the same time grammar is trained (degrees of comparison). The teacher should attract the pupils' attention to different ways of building comparative and superlative degree of adjectives - with the help of suffixes or additional words. Here the norms of grammar are deliberately violated. The ironic message is based on absurdity: the main character is getting more and more entangled in the chain of strange events, and the changes in the grammar of the language are reflecting the absurdity of the situation.

\section{$\underline{\text { Situation } 3}$}

In the process of all the transformations that are happening to her, Alice comes across a mysterious key. The girl is sure it should lead to further adventure, which acquires the image of a beautiful garden So, she is trying to find the door to this wonderful garden.

Alice: "If there is a key there must be a door."

Such transformation is similar to a famous quotation "If there is a will there is a way". The play is full of such remarks, placed in the text to emphasize the difference between our world and the one created by the author. Everything is turned upside down, even expressions like this one.

The idea of concentrating the pupils' attention on this saying is introducing them to English phraseology, comparing proverbs in the English language and their mother tongue, disclosing the meanings.

\section{Situation 4}

Alice and her friends are soaking wet after swimming in the sea of tears, which Alice had produced while being a giant. They feel cold and do not know how to warm up. The Mouse comes up 
with an idea: it is going to give a talk that is supposed to make Alice and the animals dry.

The Mouse: "Sit down, all of you, and listen to me! I'LL soon make you dry enough! 'Ahem!' Are you all ready? This is the driest thing I know."

Word play, which is used here, is very typical of the English language, rich in homonyms. The word "dry" means firstly "not wet" and secondly "cool", and both meanings can be applied in this situation. The interconnection between the literal and the figurative meaning is shown clearly, which gives actors an opportunity to enrich their vocabulary. At the same time it is desirable to concentrate on the ironic effect, which the phrase acquires by combing the two meanings of the word.

\section{$\underline{\text { Situation } 5}$}

In the same speech of The Mouse there is another detail.

The Mouse: "...Silence! William the Conqueror, whose cause was favoured by the pope..."

This phrase is an example of an unnecessary complicated official language, and that is an extremely important issue in terms of culture and national history: "Alice in Wonderland" was written in Victorian age, when children heard this language at school. Now this style is viewed with a degree of irony, when used in simple conversation.

The purpose is to introduce the learners to the topic of using various styles and the necessity to choose the necessary stylistic register in an appropriate situation.

\section{$\underline{\text { Situation } 6}$}

In the dialogue between Alice and the Caterpillar some phrases have a double meaning. For example, in the phrase "I can't explain myself, because I'm not myself" the meaning of the collocation "explain oneself" (which means "explain something clearly") is transformed into literal meaning of both words, taken separately. So the sense is different - "I can't say anything definite about my identity".

Here we deal with word play, which creates an additional ironic effect and corresponds to the situation in the story, which is getting more and more confusing.

In this connection it might be useful to point out ambiguity, which is present in the story and created by various expressive means and stylistic devices. Irony is one of the linguistic manipulations that is based on ambiguity.

\section{Situation 7}

Another example of word play, widely used by the characters, is the usage of the word "see". Alice starts her talk with the words "Well, you see..." and is interrupted. The Caterpillar says "I don't see", which has two meanings: to see in literal meaning and in collocation which can be replaced by "I don't understand".

Again, the word play contributes to the atmosphere: strange events and ridiculous behaviour of the characters become even more vivid.

\section{$\underline{\text { Situation } 8}$}

The same phenomenon can be recognized not only in the dialogue between Alice and The Caterpillar, but also in the "tea party", when Alice, the Madhatter and the Mouse start discussing another character: "He [The Time] won't stand beating" (beating as "hitting" and as "striking of the clock"). The Time is a living creature, who cannot bear being beaten; on the other hand, time is connected with the clock and counting minutes and hours. Again, we deal with ambiguity and the ironic usage of word meanings.

Another example in the same dialogue is a phrase "They lived on treacle", taken from a story about animals, who consumed the food that was under their feet ("live on" in literal meaning and "to survive by eating something"). Contrasting the two senses of a word is not only an individual characteristics of the author (Lewis Caroll), but a peculiarity of the English communicative style and one of ironic mechanisms, frequently used in discourse. This is ironic word play, which adds a note of paradox to the sense and provides amusement to the audience.

\section{$\underline{\text { Situation } 9}$}

Some logical nonsense, typical for the British mentality, can be found in the text of the play: The Madhatter's watch shows date, not the time; The Queen orders the Madhatter not to be nervous otherwise he'll be executed (and this threat cannot calm down anybody, but, on the contrary, is sure to make a person even more nervous). These are all paradoxes which create an atmosphere of absurdity and ensure the ironic perception of the play.

At this point it is desirable to discuss the roots, origins and mechanisms of British humour and irony, which are often based on absurdity, contrasting meanings, paradoxes. For the students of the English language it is important to recognize such notions and humour and irony in discourse and know the ways they function and the role they perform in speech.

While reproducing the text, the young actors learn more than only linguistic material - they get acquainted with English cultural and communicative values and the style of communication. 


\section{Discussion}

Staging the play is the most exciting moment in the course of drama, it is a sort of exam which reveals all the acquired knowledge and skills - both artistic and linguistic ones. It should be noted that the exam is not only for young actors but for the teacher as well, while the jury is represented by the audience of spectators. This form of control is more serious than an ordinary language test but the pupils are more enthusiastic about it since they perceive it not as an exam but as a play.

During the performance the children display everything they have learned - the knowledge of the text, the ability to react and interact, artistic skills, creativity etc. The young actors understand that they have only one chance to be at their best and usually make the most of it. The role of the teacher here is to support and encourage, inspire and praise.

A very important part of the drama class is the discussion after the play is over and the teacher estimates the work of every actor. Here a great deal of encouragement and advice is required from the teacher concerning the drama part. As for the language, it is desirable to go through mistakes, made during the play (including phonetics, grammar and lexis) and correct them while the children still remember their performing and are under the impression of just being on the stage in front of the audience.

On the whole, the performance itself is the shortest part of the drama course, but it is the most important one, for it reveals the results and allows to set new goals and tasks.

\section{Conclusions}

Combining learning a language, drama classes and playing on the stage opens vast perspectives in terms of acquiring knowledge as well as developing personality.

First, pupils set themselves an aim for realizing when they start working as a team. It contributes to their communicative skills. Drama classes combined with teaching a foreign language pose challenges for both students/pupils and teachers and open vast perspectives in terms of developing creativity as well as linguistic skills.

Second, to convey a certain meaning and emotions, young actors feel free to disclose their hidden talents and reveal their personalities, which is very important from psychological point of view.

Third, they master a considerable amount of lexis and grammar. The language material (which corresponds to the age and level of knowledge) is easily absorbed and remembered in the process of role-play. Learning irony and wordplay through staging a performance is an interesting and useful method, which combines different approaches: analyzing irony and ironic wordplay as a text category and viewing them as a discoursive phenomenon.

Finally, the course culminates in creating a certain product - a staged performance, which is a contribution to general education, background and aesthetical well-being. Being involved in a play students/pupils feel more relaxed and absorb information easily. The aim of staging a play makes the actors motivated. This creates a positive approach and inspires students/pupils acquire the language material and skills.

\section{Acknowledgments}

We express our gratitude to the pupils of secondary school № 1279 of Moscow, Russia, who took part in staging the play "Alice in Wonderland" and proved to be most creative and talented actors.

We thank the organizing committee of Ireland International Conference on Education (IICE-2019) for giving an opportunity to present the report and discuss the suggested approach with colleagues.

\section{References}

[1] Fox, K., Watching the English. The hidden rules of English behavior, Hodder and Stoughton, London, 2005.

[2] Hutcheon, L. Irony's Edge. The Theory and Politics of Irony, New York: Routledge, 2005.

[3] Beliaeva, V., Learning a foreign language through theatre: secondary school experience, Inted 2019 proceedings,

[https://library.iated.org/view/BELIAEVA2019LEA], 2019.

[4] Gałązka, A., Drama in education for sustainable development, Inted 2017 proceedings, [https://library.iated.org/view/GALAZKA2017DRA], 2017.

[5] Rooney, C., Coleman, M., Applying lessons from drama and stage in formal communication training: strategies to overcome oral communication apprehension for business students, Inted 2019 proceedings, [https://library.iated.org/view/ROONEY2019APP], 2019.

[6] Yaroslavova, E., Shraiber, E., Drama and theatre in foreign language teaching at the University level, Inted 2016 proceedings, [https://library.iated.org/view/YAROSLAVOVA2016DRA ], 2016.

[7] Tovstonogov, G., The mirror of the stage, Textbook, Planeta muzyki, 2018. (In Russian).

[8] Carroll L., Alice's adventures in wonderland, Usborne Publishing, 2015. 